\title{
Pilot Induced Oscillation Mitigation for Unmanned Aircraft Systems: An Adaptive Control Allocation Approach
}

\author{
S. S. Tohidi ${ }^{1}$, Y. Yildiz ${ }^{1}$, and I. Kolmanovsky ${ }^{2}$
}

\begin{abstract}
This paper proposes an adaptive control allocation method that can make unmanned aircraft systems recover from pilot induced oscillations. Pilot induced oscillations are undesired oscillations due to an unintentional and detrimental coupling between the aircraft and the pilot. These oscillations may be instigated due to disturbances, aggressive maneuvers and actuator saturation. Different from manned aircraft, pilot induced oscillations in unmanned aircraft systems are harder to handle due to communication time delays between the operator and the aircraft. The task of a conventional control allocator is to distribute the control effort among redundant actuators to realize a desired virtual control input. When actuators rate saturate, the difference between the desired and the achieved virtual control input introduces an effective time delay to the system dynamics which causes oscillations. In the proposed approach, instead of minimizing the error between the desired and achieved virtual control inputs, the derivative of this error is minimized which eliminates the introduced time delay effect and damps undesired oscillations. Differently from earlier works conducted by the authors, in this work, the proposed pilot induced oscillation mitigation methodology is developed for systems with parametric uncertainty. In the simulations, it is demonstrated that the proposed approach successfully damps pilot induced oscillations that are instigated by a high gain pilot command.
\end{abstract}

\section{INTRODUCTION}

A pilot induced oscillation (PIO) occurs due to an abnormal interaction between the pilot and the aircraft which leads to undesirable oscillations or even instability [1]. Actuator rate saturation is observed in almost all PIO cases, which introduces an effective time delay, or phase lag, to the system and thereby acts as a destabilizing factor (See Fig. 1). An analysis of the effect of rate saturation on the formation of PIOs can be found in [3].

UAS PIOs are harder to handle compared to PIOs being observed in manned aircraft due to the inevitable communication time delays between the UAS operator and the aircraft. Depending on the mission type, these delays can be uncertain and time-varying and have the potential to instigate PIOs and sometimes even cause the loss of the aircraft [4]-[6].

In order to recover from a PIO, the phase shift between the commanded and actual signals should be minimized. Several successful approaches are proposed in the literature to achieve this goal. One of these approaches is called the differentiate-limit-integrate (DLI) method [7]-[12], where a

\footnotetext{
${ }^{1}$ Seyed Shahabaldin Tohidi and Yildiray Yildiz are with Faculty of Mechanical Engineering, Bilkent University, Cankaya, Ankara 06800, Turkey shahabaldin@bilkent.edu.tr, yyildiz@bilkent.edu.tr

${ }^{2}$ Ilya Kolmanovsky is with the Department of Aerospace Engineering, University of Michigan, Ann Arbor, MI 48109, USA i lya @umi ch . edu
}

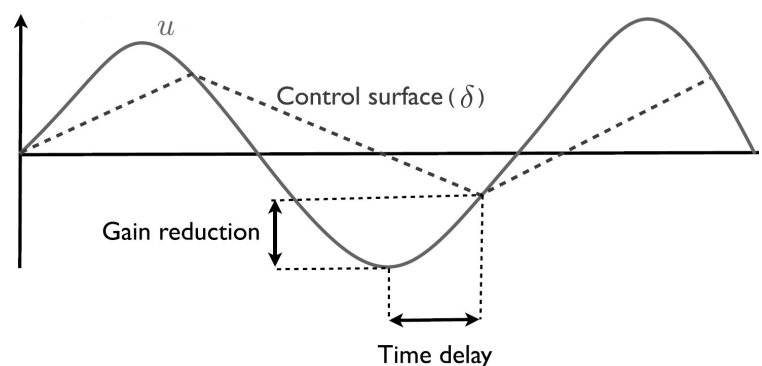

Fig. 1: Input $u$ and output $\delta$ of a rate-saturated actuator [2].

software rate limiter is placed between the commanded signal and the input signal to the actuators. Although this is an effective method to recover from a PIO, it may introduce a bias between the desired and achieved control commands and its performance may be degraded in presence of noise. However, these deficiencies can be eliminated using the methods provided in [8], [9], [11], [12]. Other methods based on manipulating the input signal such as nonlinear adaptive filters to attenuate the stick gain [13] and phase compensating filters [14]-[16] also exist in the literature.

Unlike the solutions discussed above, which are designed for single input single output systems, a method called "Control Allocation to recover from Pilot Induced Oscillations" (CAPIO) [17], [18], which was proposed by the authors, can be employed to mitigate PIOs for multi input multi output systems with redundant actuators. The main idea behind CAPIO is that unlike conventional control allocation methods, instead of minimizing the error between the desired and achieved commanded signals, the derivative of the error is minimized, which eliminates the introduction of the phase shift. The stability of a closed loop control system utilizing CAPIO is rigorously analyzed in [2], and [19] and successful human-in-the-loop experimental results with real pilots are reported in [20]. In this paper, we develop an adaptive version of CAPIO, termed "Adaptive CAPIO", inspired by our recent work on adaptive control allocation, reported in [21] and [22], and apply it to UAS recovery from PIOs.

Control allocation methods can be categorized into three main categories: pseudo inverse based methods ( [23]-[25]), optimization based methods ( [17], [26]-[28]), and dynamic control allocation ( [21], [22], [29]-[31]). A survey of control allocation can be found in [34], where the use of control allocation to address actuator faults is highlighted. Also, in [32], a control allocation method is proposed where an unknown input observer is applied to identify actuator and effector faults. The method given in [21] and [22], and used 
as the basis for the development of the proposed Adaptive CAPIO in this paper, can also be used to compensate actuator faults, without the need for fault identification.

This paper is organized as follows. Section II presents control allocation problem statement. The adaptive control allocation is presented in Section III. Section IV presents the adaptive CAPIO. Controller design is presented in section V. Section VI illustrates the effectiveness of the proposed method in the simulation environment. Finally, Section VII concludes the paper.

\section{CONTROL ALLOCATION PROBLEM STATEMENT}

Consider the following plant dynamics

$$
\dot{x}=A x+B_{u} u,
$$

where $A \in R^{n \times n}$ is the known state matrix, $B_{u} \in R^{n \times m}$ is the known control input matrix, $x \in R^{n}$ is the system state vector, and $u=\left[u_{1}, u_{2}, \ldots, u_{m}\right]^{T} \in R^{m}$ is the vector of actuator commands with amplitude limits $u_{i} \in\left[-u_{\max _{i}}, u_{\max _{i}}\right]$ and rate limits $\dot{u}_{i} \in\left[-\dot{u}_{\text {max }_{i}}, \dot{u}_{\text {max }_{i}}\right]$ for $i=1, \ldots, m$.

In over-actuated systems, the control input matrix is rank deficient that is $\operatorname{Rank}\left(B_{u}\right)=r<m$. Consequently, $B_{u}$ can always be decomposed into $B_{u}=B_{v} B$, where $B \in R^{r \times m}$ and $B_{v} \in R^{n \times r}$, such that $B_{v}$ is a full column rank matrix i.e. $\operatorname{Rank}\left(B_{v}\right)=r$. The reason for the decomposition of $B_{u}$ is to represent the redundancy in a form that can be exploited in control allocation.

To model the actuator uncertainty, a diagonal matrix $\Lambda \in$ $R^{m \times m}$ with uncertain positive elements is added to the system dynamics as follows

$$
\dot{x}=A x+B_{v} B \Lambda u .
$$

The control allocation problem is to achieve $B \Lambda u=v$, where $v \in R^{r}$ denotes the virtual control input produced by the controller. Thus, the plant "seen" by the controller, with ideal control allocation, can be written as

$$
\dot{x}=A x+B_{v} v \text {. }
$$

Since $\Lambda$ is unknown, conventional control allocation methods, which require the exact knowledge of the control input matrix $B_{u}$, do not apply. In addition, it is required that matrix identification methods are not used since they require persistence of excitation which is hard to realize in real applications.

\section{ADAPTIVE CONTROL ALLOCATION}

The goal of the control allocation is to distribute virtual control signals among redundant actuators and minimize the difference between the achieved and the desired virtual control inputs. Towards this end, we first transform the control allocation problem into a conventional model reference adaptive control problem and then develop the corresponding adaptive laws.

Consider the following dynamics

$$
\dot{y}=A_{m} y+B \Lambda u-v,
$$

where $A_{m} \in R^{r \times r}$ is a stable matrix. A reference model is given as

$$
\dot{y}_{m}=A_{m} y_{m}
$$

Defining the control input as a mapping from $v$ to $u$,

$$
u=\theta_{v}^{T} v
$$

where $\theta_{v} \in R^{r \times m}$ represents the adaptive parameter matrix to be determined, and substituting (6) into (4), we obtain

$$
\dot{y}=A_{m} y+\left(B \Lambda \theta_{v}^{T}-I_{r}\right) v,
$$

where $I_{r}$ is an identity matrix of dimension $r \times r$.

It is assumed that there exists a $\theta_{v}^{*}$ such that

$$
B \Lambda \theta_{v}^{* T}=I_{r} .
$$

Defining an error as $e \equiv y-y_{m}$ and subtracting (5) from (7), it follows that

$$
\dot{e}=A_{m} e+B \Lambda \tilde{\theta}_{v}^{T} v
$$

where $\tilde{\theta}_{v}=\theta_{v}-\theta_{v}^{*}$. Consider a Lyapunov function candidate,

$$
V=e^{T} P e+\operatorname{tr}\left(\tilde{\theta}_{v}^{T} \Gamma_{\theta}^{-1} \tilde{\theta}_{v} \Lambda\right),
$$

where $\Gamma_{\theta}=\Gamma_{\theta}^{T}=\gamma_{\theta} I_{r}, \gamma_{\theta}>0, t r$ refers to the trace operation and $P$ is the positive definite symmetric matrix solution of the Lyapunov equation $A_{m}^{T} P+P A_{m}=-Q$, where $Q$ is a symmetric positive definite matrix. The derivative of the Lyapunov function candidate can be calculated as

$$
\begin{aligned}
\dot{V} & =e^{T}\left(A_{m} P+P A_{m}\right) e+2 e^{T} P B \Lambda \tilde{\theta}_{v}^{T} v+2 \operatorname{tr}\left(\tilde{\theta}_{v}^{T} \Gamma_{\theta}^{-1} \dot{\tilde{\theta}}_{v} \Lambda\right) \\
& =-e^{T} Q e+2 e^{T} P B \Lambda \tilde{\theta}_{v}^{T} v+2 \operatorname{tr}\left(\tilde{\theta}_{v}^{T} \Gamma_{\theta}^{-1} \dot{\tilde{\theta}}_{v} \Lambda\right) \\
& =-e^{T} Q e+2 \operatorname{tr}\left(\tilde{\theta}_{v}^{T}\left(v e^{T} P B+\Gamma_{\theta}^{-1} \dot{\tilde{\theta}}_{v}\right) \Lambda\right) .
\end{aligned}
$$

Using the following adaptive law,

$$
\dot{\theta}_{v}=\Gamma_{\theta} \operatorname{Proj}\left(\theta_{v},-v e^{T} P B\right),
$$

where "Proj" refers to the projection operator [35], and using the property of the projection operator [35], that is

$$
\operatorname{tr}\left(\tilde{\theta}_{v}^{T}\left(-Y+\operatorname{Proj}\left(\theta_{v}, Y\right)\right) \Lambda\right) \leq 0,
$$

where $Y=-v e^{T} P B$, yields $\dot{V} \leq 0$. Therefore, assuming that $v$ is bounded, all the signals in the adaptive control allocation system are bounded. Additional details, such as the calculation of the bounds on the error $e$, can be found in [21].

Remark 1. The boundedness assumption of $v$ will be justified in the controller design section.

\section{ADAPTIVE CAPIO}

Motivated by the approach of matching the phase of $B \Lambda u$ and $v$, consider the following dynamics

$$
\dot{y}=A_{m} y+B \Lambda \dot{u}-\dot{v},
$$

where $A_{m} \in R^{r \times r}$ is a stable matrix. Defining the derivative of the actuator commands as a mapping from $\dot{v}$ to $\dot{u}$ as

$$
\dot{u}=\varphi_{v}^{T} \dot{v}
$$


where $\varphi_{v} \in R^{r \times m}$ represents the adaptive parameter matrix to be determined, and substituting (15) into (14), we obtain that

$$
\dot{y}=A_{m} y+\left(B \Lambda \varphi_{v}^{T}-I_{r}\right) \dot{v}
$$

where $I_{r}$ is an identity matrix of dimension $r \times r$. Note that once $\dot{u}$ is determined as in (15), the actuator commands are determined by integrating $\dot{u}$. It is assumed that there exists a $\varphi_{v}^{*}$ such that

$$
B \Lambda \varphi_{v}^{* T}=I_{r}
$$

Defining $\varphi_{v}^{T}=\varphi_{v}^{* T}+\tilde{\varphi}_{v}^{T}$, where $\tilde{\varphi}_{v}^{T}$ is the deviation of $\varphi_{v}^{T}$ from its ideal value, equation (16) can be rewritten as

$$
\dot{y}=A_{m} y+B \Lambda \tilde{\varphi}_{v}^{T} \dot{v} .
$$

Defining an error $e=y-y_{m}$, where $y_{m}$ is determined by the reference model dynamics (5), and taking its derivative using (5) and (18), it follows that

$$
\dot{e}=A_{m} e+B \Lambda \tilde{\varphi}_{v}^{T} \dot{v} .
$$

Let $\Gamma_{\varphi}=\Gamma_{\varphi}^{T}=\gamma I_{r} \in R^{r \times r}$, where $\gamma$ is a positive scalar, and consider a Lyapunov function candidate,

$$
V_{1}=e^{T} P e+\operatorname{tr}\left(\tilde{\varphi}_{v}^{T} \Gamma_{\varphi}^{-1} \tilde{\varphi}_{v} \Lambda\right),
$$

where $\operatorname{tr}$ refers to the trace operation and $P$ is the positive definite symmetric matrix solution of the Lyapunov equation. The derivative of the Lyapunov function candidate can be calculated as

$$
\begin{aligned}
\dot{V}_{1} & =e^{T}\left(A_{m} P+P A_{m}\right) e+2 e^{T} P B \Lambda \tilde{\varphi}_{v}^{T} \dot{v}+2 \operatorname{tr}\left(\tilde{\varphi}_{v}^{T} \Gamma_{\varphi}^{-1} \dot{\tilde{\varphi}}_{v} \Lambda\right) \\
& =-e^{T} Q e+2 e^{T} P B \Lambda \tilde{\varphi}_{v}^{T} \dot{v}+2 \operatorname{tr}\left(\tilde{\varphi}_{v}^{T} \Gamma_{\varphi}^{-1} \dot{\tilde{\varphi}}_{v} \Lambda\right) \\
& =-e^{T} Q e+2 \operatorname{tr}\left(\tilde{\varphi}_{v}^{T}\left(\dot{v} e^{T} P B+\Gamma_{\varphi}^{-1} \dot{\tilde{\varphi}}_{v}\right) \Lambda\right) .
\end{aligned}
$$

Using the following adaptive law

$$
\dot{\varphi}_{v}=\Gamma_{\varphi} \operatorname{Proj}\left(\varphi_{v},-\dot{v} e^{T} P B\right)
$$

where "Proj" refers to the projection operator [35], and using the property of the projection operator [35], it follows that $\dot{V}_{1} \leq 0$. Therefore, assuming that $\dot{v}$ is bounded, all system signals can be shown to be bounded.

Remark 2. The boundedness assumption for $\dot{v}$ will be justified in the controller design section.

Remark 3. Adaptive control allocator developed in Section III is the default mode for the control allocation. Adaptive CAPIO developed in this section will be activated once a PIO is detected and will be switched off once PIO is over.

\section{CONTROLLER DESIGN}

A block diagram depicting the overall closed loop UAS system is given in Fig. 2. In the previous sections, the design of the control allocator was explained. In this section, the design of a suitable controller is provided. It is noted that only one of the possible controllers is explained in this section. Other controllers suitable for the application can also be used. In (2), the disturbance was not included in plant dynamics since the focus was on control allocation development. In this section, we design the controller in the presence of an additive disturbance. Consider the following plant dynamics

$$
\dot{x}=A x+B_{v}(B \Lambda u+\rho)=A x+B_{v} B \Lambda u+B_{v} \rho,
$$

where $\rho \in R^{r}$ is an additive disturbance with known upper bound $R$. By utilizing the allocation law (6), and substituting $\tilde{\theta}_{v}=\theta_{v}-\theta_{v}^{*}$, we have

$$
\begin{aligned}
\dot{x} & =A x+B_{v} B \Lambda \theta_{v}^{T} v+B_{v} \rho \\
& =A x+B_{v}\left(B \Lambda \theta_{v}^{* T}+B \Lambda \tilde{\theta}_{v}^{T}\right) v+B_{v} \rho .
\end{aligned}
$$

Substituting the ideal value of $\theta_{v}^{*}$, (using $B \Lambda \theta_{v}^{*^{T}}=I$ ), we obtain that

$$
\dot{x}=A x+B_{v}\left(I+B \Lambda \tilde{\theta}_{v}^{T}\right) v+B_{v} \rho .
$$

Since the projection algorithm is used in the adaptive laws for the control allocation, we know that $\tilde{\theta}_{v}$ is bounded, regardless of any stability condition. Defining $F(t) \equiv B \Lambda \tilde{\theta}_{v}^{T}$, (25) can be rewritten as

$$
\dot{x}=A x+B_{v}(I+F(t)) v+B_{v} \rho,
$$

where $F(t) \in R^{r \times r}$ is a matrix with bounded elements.

Lemma 1. There exists a constant $\bar{F}$, which can be computed explicitly, such that $\|F(t)\| \leq \bar{F}, \forall t \in R_{\geq 0}$.

Proof. Proof of the lemma can be found in [21].

Assume that (26) can be decomposed into two subsystems given as

$$
\begin{aligned}
& \dot{x}_{1}=A_{1} x_{1}+A_{2} x_{2} \\
& \dot{x}_{2}=A_{3} x_{1}+A_{4} x_{2}+B_{v}^{\prime}(I+F(t)) v+B_{v}^{\prime} \rho,
\end{aligned}
$$

where $x_{1} \in R^{n-r}$ and $x_{2} \in R^{r}$. Assuming that $B_{v}^{\prime} \in R^{r \times r}$ is invertible, and considering that (28) is a square system, a sliding mode controller can be designed for the control of the system given in (28) [36]. Assuming a stable $A_{1}$, showing the boundedness of the state vector $x_{2}$ will be enough to ensure the boundedness of the state vector $x_{1}$.

Remark 4. The assumptions given above about $B_{v}$ and $A_{1}$ hold true in several aerospace applications. These assumptions will be checked in a realistic implementation scenario in the simulations section.

Each individual scalar equation in (28) can be written as

$$
\dot{x}_{2 i}=h_{i}(x)+\sum_{j=1}^{r} b_{i j}(x) v_{j} \quad i=1, \ldots, r .
$$

where $b_{i j}(x)$ and $h_{i}(x)$ are unknown because of the terms $F(t)$ and $\rho$, respectively. Denote an estimate for $h_{i}(x)$ as $\hat{h}_{i}(x)$, the estimation error is bounded as $|\hat{h}(x)-h(x)| \mid \leq R$. Defining $s_{i} \equiv x_{2 i}-x_{2 d_{i}}$, where $x_{2 d_{i}}$ is the desired trajectory for $x_{2 i}$, it can be shown [36] that the following control input satisfies the sliding conditions

$$
v=B_{v}^{\prime-1}\left(x_{2 d}-h(x)-k \operatorname{sgn}(s)\right),
$$

where $x_{2 d} \in R^{r}, h(x) \in R^{r}$ and $k \operatorname{sgn}(s) \in R^{r}$ is a vector consisting of components $k_{i} \operatorname{sgn}\left(s_{i}\right)$. It is noted that $k_{i} \in R$ 
must be chosen such that satisfies the sliding condition given as $\frac{1}{2} \frac{d}{d t} s_{i}^{2} \leq-\eta_{i}\left|s_{i}\right|$, with $\eta_{i} \in R_{>0}$, which yields

$$
(1-\bar{F}) k_{i}+\sum_{j \neq i} \bar{F} k_{j}=R+\sum_{j=1}^{r} \bar{F}\left|x_{2 d_{i}}-\hat{h}_{j}\right|+\eta_{i}, i=1, \ldots, r
$$

To avoid the chattering, as an undesirable result of the controller discontinuity, a typical approach is to smooth out the discontinuity by changing the term $\operatorname{sgn}(s)$ in (30) to $\operatorname{sat}(s / \Phi)$ as:

$$
v=B_{v}^{\prime-1}\left(x_{2 d}-h(x)+k \operatorname{sat}(s / \Phi)\right),
$$

where $\Phi$ is the boundary layer thickness and sat(.) is the saturation function. The sliding mode controller (32) guarantees that the boundary layer is attractive and invariant.

It is shown above that the state vector $x_{2}$ is bounded. Using (27) and the assumption of a stable $A_{1}$, it is concluded that $x_{1}$ is also bounded. Since $x_{1}$ and $x_{2}$ are bounded, $h(x)=A_{3} x_{1}+A_{4} x_{2}$ is bounded and therefore $v$ can be shown to be bounded using (32). Boundedness of $x_{1}, x_{2}, F(t), v$ and $\rho$ ensures that $\dot{x}_{1}$ and $\dot{x}_{2}$ are bounded. Also, sat(.) is a continuous bounded function. Therefore, $\dot{v}$ is bounded.

\section{APPLICATION EXAMPLE}

\section{A. ADMIRE MODEL}

The Aerodata Model in Research Environment (ADMIRE) which is an over-actuated aircraft model is used as a UAS model to demonstrate the effectiveness of the adaptive control allocation and adaptive CAPIO in the presence of parametric uncertainties. The ADMIRE aircraft model at Mach 0.22 and altitude $3000 \mathrm{~m}$ is provided in [17] and [26], which is also given below:

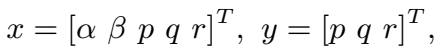

$$
\begin{aligned}
& u=\left[\begin{array}{llll}
u_{c} & u_{r e} & u_{l e} & u_{r}
\end{array}\right]^{T}, \delta=\left[\begin{array}{llll}
\delta_{c} & \delta_{r e} & \delta_{l e} & \delta_{r}
\end{array}\right]^{T}, \\
& {\left[\begin{array}{c}
\dot{x} \\
\dot{\delta}
\end{array}\right]=\left[\begin{array}{cc}
A & B \\
0 & -B_{\delta}
\end{array}\right]\left[\begin{array}{l}
x \\
\delta
\end{array}\right]+\left[\begin{array}{c}
0 \\
B_{\delta}
\end{array}\right] u,}
\end{aligned}
$$

where $\alpha, \beta, p, q$ and $r$ are the angle of attack, sideslip angle, roll rate, pitch rate and yaw rate, respectively. The state $\delta$ is augmented to the system to represent the actuator dynamics. Actuators are considered to have first order dynamics. $u$ and $\delta$ represent the commanded and actual control surface deflection vectors, where the control surfaces are the canard wings, right and left elevons and the rudder. The position and rate limits of the control surfaces are given as $\delta_{c}, \delta_{r e}, \delta_{l e}, \delta_{r} \in[-45,45] \times \frac{\pi}{180}(\mathrm{rad})$ and $\dot{\delta}_{c}, \dot{\delta}_{r e}, \dot{\delta}_{l e}, \dot{\delta}_{r} \in$ $[-70,70] \times \frac{\pi}{180}(\mathrm{rad} / \mathrm{sec})$.

In order to make the model suitable for control allocation implementation, the actuator dynamics and the control surfaces' influence on derivatives of the first two states i.e. $\dot{\alpha}$ and $\dot{\beta}$ are neglected. Thus, control surfaces are considered as pure moment generators. In addition, to represent actuator uncertainty, a diagonal matrix $\Lambda$ is introduced. With these assumptions, the system can be written as

$$
\dot{x}=A x+B_{u} \Lambda u=A x+B_{v} v, \quad B_{v}=\left[0_{3 \times 2}, I_{3 \times 3}\right]^{T} .
$$

State and control matrices are given as:

$$
\begin{gathered}
A=\left[\begin{array}{ccccc}
-0.5432 & 0.0137 & 0 & 0.9778 & 0 \\
0 & -0.1179 & 0.2215 & 0 & -0.9661 \\
0 & -10.5123 & -0.9967 & 0 & 0.6176 \\
2.6221 & -0.0030 & 0 & -0.5057 & 0 \\
0 & 0.7075 & -0.0939 & 0 & -0.2127
\end{array}\right] \\
B=\left[\begin{array}{cccc}
0 & -4.2423 & 4.2423 & 1.4871 \\
1.6532 & -1.2735 & -1.2735 & 0.0024 \\
0 & -0.2805 & 0.2805 & -0.8823
\end{array}\right] .
\end{gathered}
$$

The system given in (34) can be decomposed into the following two subsystems:

$$
\begin{aligned}
{\left[\begin{array}{l}
\dot{\alpha} \\
\dot{\beta}
\end{array}\right] } & =\left[\begin{array}{cc}
-0.5432 & 0.0137 \\
0 & -0.1179
\end{array}\right]\left[\begin{array}{l}
\alpha \\
\beta
\end{array}\right] \\
& +\left[\begin{array}{ccc}
0 & 0.9778 & 0 \\
0.2215 & 0 & -0.9661
\end{array}\right]\left[\begin{array}{l}
p \\
q \\
r
\end{array}\right],
\end{aligned}
$$

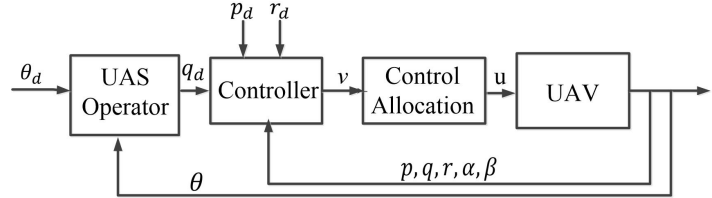

Fig. 2: Block diagram of the pilot in the loop system.

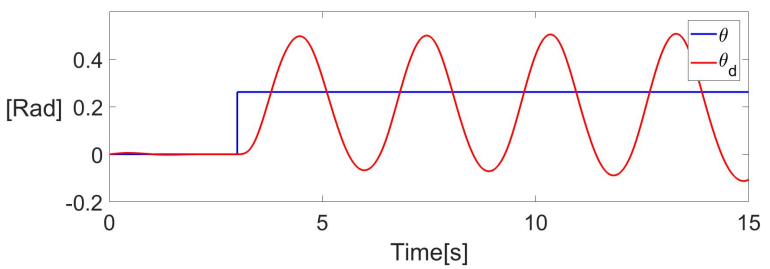

Fig. 3: UAS operator's aggressive maneuver, represented by a high gain operator model, results in a PIO in the pitch axis.

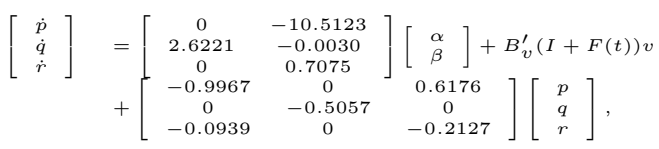

where $B_{v}^{\prime}$ is the identity matrix. It is noted that the state matrix of the first subsystem is stable and $B_{v}^{\prime}$ is invertible. Therefore, the assumptions used to develop the outer loop controller, given after (27) and (28) and discussed at Remark 4, are hold justified. The matrix $A_{m}$ is selected as $A_{m}=\operatorname{diag}(-0.2,-0.1,-0.1)$ which is a stable matrix.

\section{B. Simulation Results}

Figure 2 shows the block diagram of the closed loop system consisting of UAS operator, controller, control allocator and UAS. A scalar gain is used to model the operator for simplicity. The desired pitch angle $\left(\theta_{d}\right)$ is being tracked by the pilot by using a stick that specifies a pitch rate reference $q_{d}$ for the controller. Desired roll rate $\left(p_{d}\right)$ and yaw rate $\left(r_{d}\right)$ are specified separately.

Figure 3 shows the oscillations in pitch angle produced after the UAS operator responds to a step pitch reference using a gain of 2.5 (see Fig. 2). This gain value corresponds to the loop gain which is large enough to create sustained oscillations. The controller also receives a pulse roll and a zero yaw references. The control signals are distributed among redundant actuators using adaptive control allocation. Figure 4 shows the states of the system. Figure 5 shows the control surface deflections where position and rate saturation of the actuators are clearly visible. These saturations are also responsible for the phase shift between the desired and the achieved pitch rates that can be observed in Fig. 4. Figure 6 shows the performance of the control allocator in terms of realizing virtual control input demands. It is seen that, although the closed loop system has marginally stable behavior, the control allocation is stable.

It is assumed that there exist a PIO detector in the system which detects the PIO and switches the Adaptive CAPIO on at $t=6 \mathrm{sec}$. To demonstrate the adaptive capabilities of the proposed method, it is assumed that extensive oscillations introduce a fault to the actuators at $t=8 \mathrm{sec}$, which reduces the actuator effectiveness to $70 \%$ for all actuators. Figure 7 shows how the PIO in the pitch axis is eliminated with the introduction of the Adaptive CAPIO at $t=6 \mathrm{sec}$. Figure 8 shows the states of the system. Figure 9 shows the control surface deflections, where the effects of actuator faults can be observed at time $t=8 \mathrm{sec}$. Figure 10 shows the performance of the control allocator in terms of realizing the desired virtual control input $v$. 


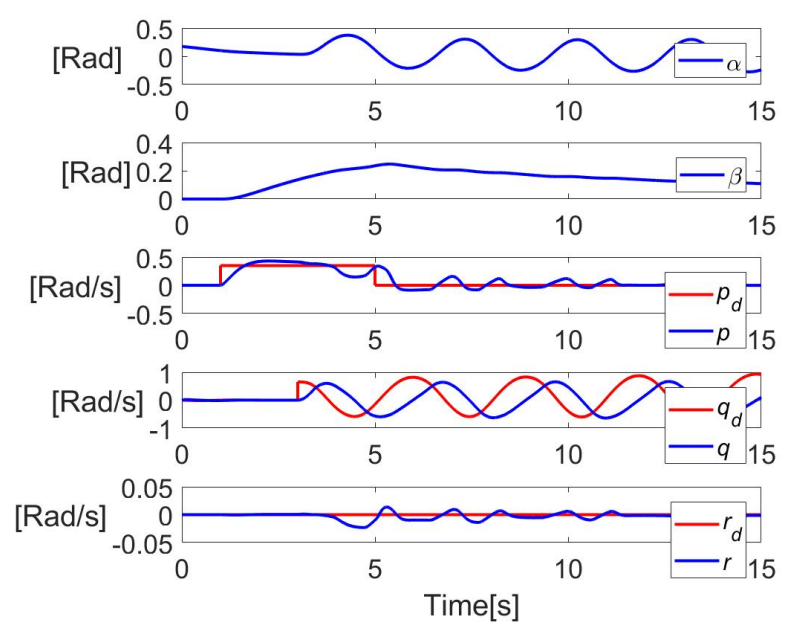

Fig. 4: System states, during a PIO, using adaptive control allocation.
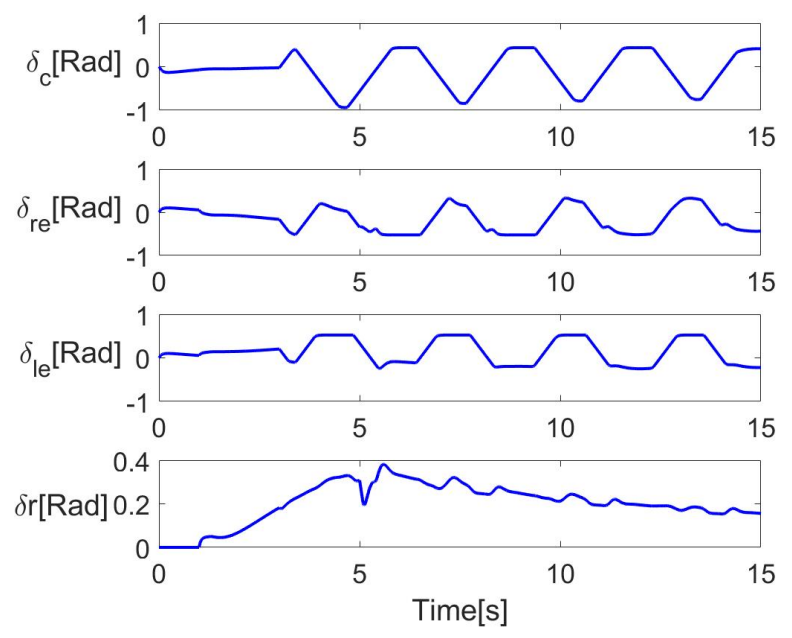

Fig. 5: Control surface deflections, using adaptive control allocation.
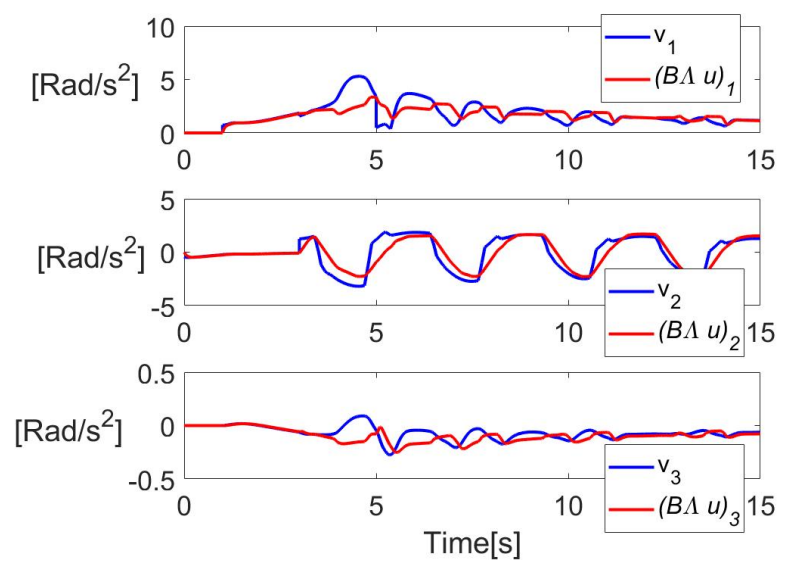

Fig. 6: Control allocation performance, using adaptive control allocation.

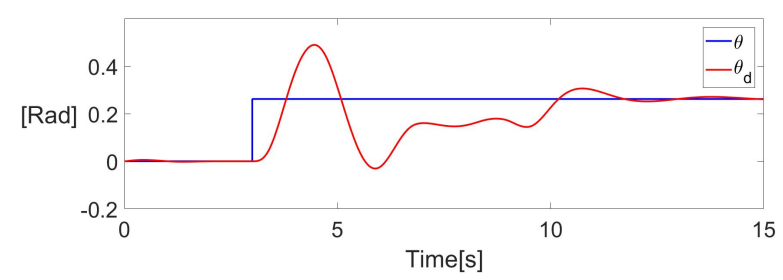

Fig. 7: PIO recovery in the pitch axis with the introduction of the Adaptive CAPIO at $t=6 \mathrm{sec}$. Adaptive CAPIO is switched off and adaptive control allocation takes its place at $t=9 \mathrm{sec}$.
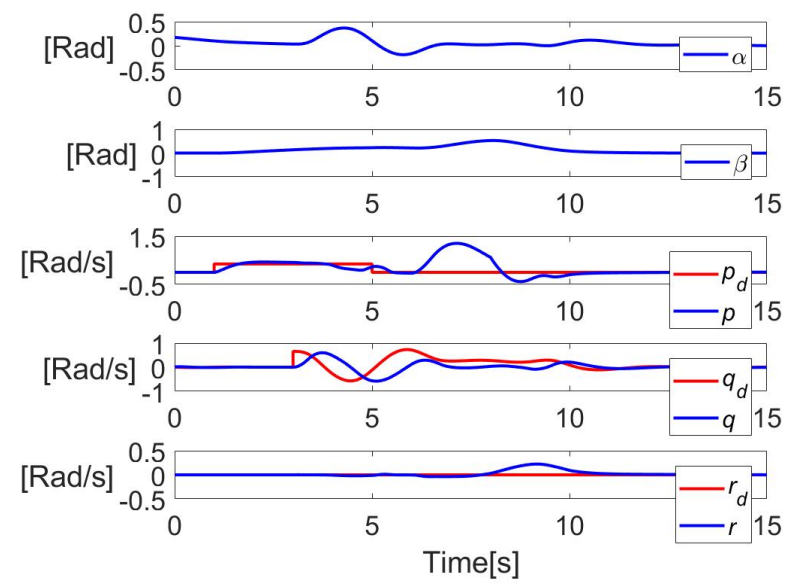

Fig. 8: System states, when adaptive CAPIO is switched on at $t=6 \mathrm{sec}$ and off at $t=9 \mathrm{sec}$.
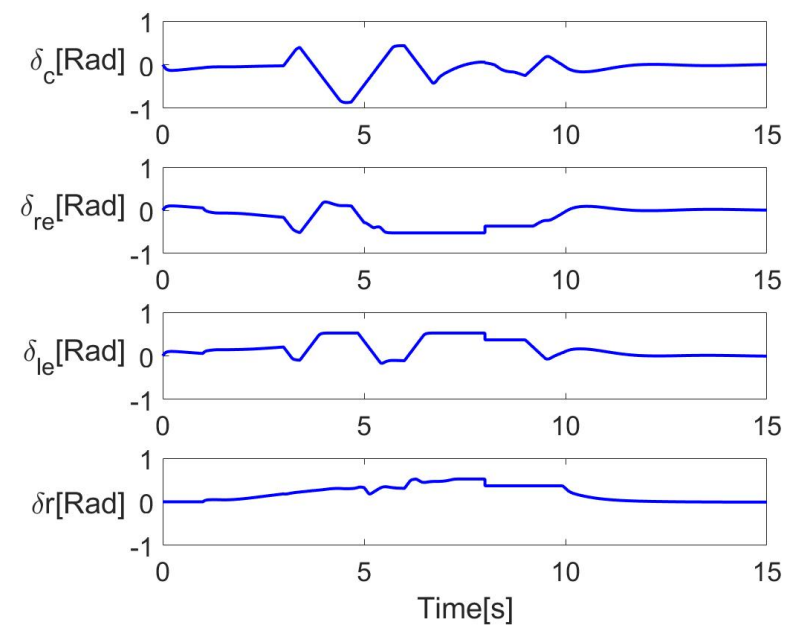

Fig. 9: Control surface deflections, when adaptive CAPIO is switched on at $t=6 \mathrm{sec}$ and off at $t=9 \mathrm{sec}$. 

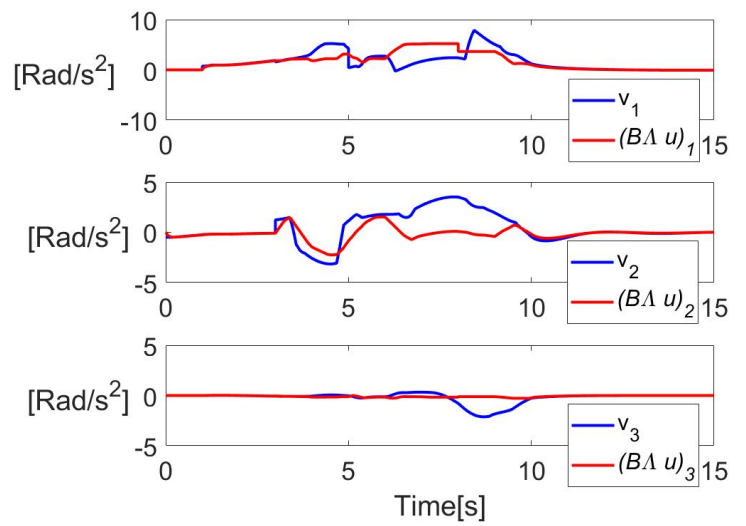

Fig. 10: Control allocation performance, when adaptive CAPIO is switched on at $t=6 \mathrm{sec}$ and off at $t=9 \mathrm{sec}$.

\section{CONCLUSIONS}

An Adaptive Control Allocation method to recover from Pilot Induced Oscillations, termed Adaptive CAPIO, is proposed in this paper. In this method, the error between the derivatives of the desired and the achieved virtual control inputs is minimized to eliminate the phase shift introduced due to actuator rate saturation. In the case of adaptive CAPIO, this is accomplished in the presence of actuator uncertainties. A scenario where a PIO is instigated by a high gain maneuver of an unmanned aircraft operator, demonstrates that the proposed control allocation method is able to dampen and eliminate the PIO in the presence of uncertain actuator effectiveness.

\section{REFERENCES}

[1] M. R. Anderson, "Pilot-Induced Oscillations Involving Multiple Nonlinearities," Journal of Guidance, Control and Dynamics, vol. 21, pp. 786-791, 1998.

[2] Y. Yildiz, and I. Kolmanovsky, "Stability Properties and CrossCoupling Performance of the Control Allocation Scheme CAPIO," Journal of Guidance, Control and Dynamics, pp. 1190-1196, 2011.

[3] D. H. Klyde, and D. G. Mitchell, "Investigating the role of rate limiting in pilot-induced oscillations," Journal of Guidance, Control and Dynamics, vol. 27, pp. 804-813, 2004.

[4] S. K. Sarrafian, "Simulator evaluation of a remotely piloted vehicle visual landing task," Journal of Guidance, Control and Dynamics, vol. 9, pp. 80-84, 1986.

[5] J. T. Hing, P. Y. Oh, "Integrating motion platforms with unmanned aerial vehicles to improve control, train pilots and minimize accidents," ASME International Design Engineering Technical Conferences and Computers and Information in Engineering Conference, pp. 867-875, 2008.

[6] J. T. Hing, P. Y. Oh, "Development of an unmanned aerial vehicle piloting system with integrated motion cueing for training and pilot evaluation," Journal of Intelligent and Robotic Systems, vol. 54, pp. 3-19, 2009.

[7] B. S. Liebst, M. J. Chapa, and D. B. Leggett, "Nonlinear prefilter to prevent pilot-induced oscillations due to actuator rate limiting," Journal of Guidance, Control and Dynamics, vol. 25, pp. 740-747, 2002.

[8] M. Chapa, "A nonlinear pre-lter to prevent departure and/or pilot induced oscillations (PIO) due to actuator rate limiting," M.S. Thesis, AFIT/GAE/ENY/99M-01, Graduate School of Engineering, Air Force Inst. of Technology (AU), Wright-Patterson AFB, OH, 1999.

[9] B. S. Liebst, M. J. Chapa, and D. B. Leggett, "Nonlinear prelter to prevent pilot-induced oscillations due to actuator rate limiting,"Journal of Guidance, Control and Dynamics, vol. 25, pp. 740747, 2002.

[10] P. Deppe, C. Chalk, and M. Shafer "Flight Evaluation Of An Aircraft With Side And Centerstick Controllers And Rate-Limited Ailerons." Advanced Technology Center, Calspan Corp., Final Rept. 8091-2, Buffalo, NY, April 1994.
[11] R. A. Hess, and S. A. Shell, "Flight control system design and rate saturating actuators," Journal of Guidance, Control and Dynamics, vol. 20, pp. 90-96, 1997.

[12] S. A. Shell, and R. A. Hess, "Robust decoupled flight control design with rate-saturating actuators," Journal of Guidance, Control and Dynamics, vol. 21, pp. 361-367, 1998.

[13] J. W. Smith, and J. W. Edwards, "Design of a nonlinear adaptive filter for suppression of shuttle pilot-induced oscillation tendencies, NASA TM-81349, April 1980.

[14] J. Koper, "An approach for compensating actuator rate saturation," Air Vehicle and Crew systems Technology Dept., Naval Air Development Center, Warminster, PA, Interim Rept. NADC-87120-60, Aug. 1987.

[15] L. Rundqwist, and R. Hillgren, "Phase Compensation of Rate Limiters in JAS 39 Gripen," AIAA, pp. 1996-3368, July 1996.

[16] D. Hanke, "Phase compensation: A means of preventing aircraft-pilot coupling caused by rate limitation," DLR - Forschungsbericht 98-15 Tech. Rep., 1998.

[17] Y. Yildiz, and I. Kolmanovsky, "A control allocation technique to recover from pilot-induced oscillations (CAPIO) due to actuator rate limiting," American Control Conference, pp. 516-523, 2010.

[18] Y. Yildiz, I. Kolmanovsky, and D. Acosta, "A control allocation system for automatic detection and compensation of phase shift due to actuator rate limiting," American Control Conference, pp. 444-449, 2011.

[19] Y. Yildiz, and I. Kolmanovsky, "Implementation of CAPIO for composite adaptive control of cross-coupled unstable aircraft," Infotech@ Aerospace, 2011.

[20] D. M. Acosta, Y. Yildiz, R. W. Craun, S. D. Beard, M. W. Leonard, G. H. Hardy, and M. Weinstein, "Piloted Evaluation of a Control Allocation Technique to Recover from Pilot-Induced Oscillations," Journal of Aircraft, vol. 52, pp. 130-140, 2015.

[21] S. S. Tohidi, Y. Yildiz, and, I. Kolmanovsky, "Fault Tolerant Control for Over-Actuated Systems: An Adaptive Correction Approach," American Control Conference, pp. 2530-2535, 2016.

[22] S. S. Tohidi, Y. Yildiz, and, I. Kolmanovsky, "Adaptive Control Allocation for Over-Actuated Systems with Actuator Saturation," IFAC, pp. 5492-5497, 2017.

[23] W. C. Durham, "Constrained Control Allocation," Journal of Guidance, Control and Dynamics, vol. 16, pp. 717-725, 1993.

[24] H. Alwi, and C. Edwards, "Fault tolerant control using sliding modes with on-line control allocation," Automatica, vol. 44, pp. 1859-1866, 2008.

[25] S. S. Tohidi, A. Khaki Sedigh, and D. Buzorgnia, "Fault tolerant control design using adaptive control allocation based on the pseudo inverse along the null space.," International Journal of Robust and Nonlinear Control, vol. 26, pp. 3541-3557, 2016.

[26] O. Harkegard, and S. T. Glad, "Resolving actuator redundancy Optimal control vs. control allocation," Automatica, vol. 41, pp. 137144, 2005.

[27] J. A. M. Petersen, and M. Bodson, "Constrained quadratic programming techniques for control allocation," IEEE Transaction on Control Systems Technology, vol. 14, pp. 91-98, 2006.

[28] A. Casavola, and E. Garone, "Fault-tolerant adaptive control allocation schemes for overactuated systems," Int. J. Robust Nonlinear Sys., vol. 20, pp. 1958-1980, Jan. 2010.

[29] L. Zaccarian, "Dynamic allocation for input redundant control systems," Automatica, vol. 45, pp. 1431-1438, 2009.

[30] Y. Luo, A. Serrani, S. Yurkovich, D. B. Doman, and M. W. Oppenheimer, "Model Predictive Dynamic Control Allocation with Actuator Dynamics," American Control Conference, pp. 1695-1700, 2004.

[31] J. Tjønnås, and T.A. Johansen, "Adaptive control allocation," Automatica, vol. 44, pp. 2754-2765, 2008.

[32] A. Cristofaro, and T. A. Johansen, "Fault tolerant control allocation using unknown input observers," Automatica, vol. 50, pp. 1891-1897, 2014.

[33] G. P. Falconí, and F. Holzapfel, "Adaptive fault tolerant control allocation for a hexacopter system," American Control Conference, pp. 6760-6766, 2016.

[34] T. A. Johansen, and T. I. Fossen, "Control allocation-a survey," Automatica, vol. 49, pp. 1087-1103, May 2013.

[35] E. Lavretsky, and T. E. Gibson, "Projection operator in adaptive systems," arXiv ePrints, arXiv:1112.4232, 2011.

[36] J. J. E. Slotine, and W. Li, Applied Nonlinear Control. Prentice-Hall, 1991. 